\title{
Focusing Magnets for HIF Based on Racetracks
}

\author{
N. N. Martovetsky, R. R. Manahan
}

This article was submitted to Applied Superconductivity Conference 2000, Virginia Beach, VA, September 17-22, 2000

Lawrence

September 11, 2000 


\section{DISCLAIMER}

This document was prepared as an account of work sponsored by an agency of the United States Government. Neither the United States Government nor the University of California nor any of their employees, makes any warranty, express or implied, or assumes any legal liability or responsibility for the accuracy, completeness, or usefulness of any information, apparatus, product, or process disclosed, or represents that its use would not infringe privately owned rights. Reference herein to any specific commercial product, process, or service by trade name, trademark, manufacturer, or otherwise, does not necessarily constitute or imply its endorsement, recommendation, or favoring by the United States Government or the University of California. The views and opinions of authors expressed herein do not necessarily state or reflect those of the United States Government or the University of California, and shall not be used for advertising or product endorsement purposes.

This is a preprint of a paper intended for publication in a journal or proceedings. Since changes may be made before publication, this preprint is made available with the understanding that it will not be cited or reproduced without the permission of the author.

This report has been reproduced directly from the best available copy.

Available electronically at http://www.doc.gov/bridge

Available for a processing fee to U.S. Department of Energy

And its contractors in paper from

U.S. Department of Energy

Office of Scientific and Technical Information

P.O. Box 62

Oak Ridge, TN 37831-0062

Telephone: (865) 576-8401

Facsimile: (865) 576-5728

E-mail: reports@adonis.osti.gov

Available for the sale to the public from

U.S. Department of Commerce

National Technical Information Service

5285 Port Royal Road

Springfield, VA 22161

Telephone: (800) 553-6847

Facsimile: (703) 605-6900

E-mail: orders@ntis.fedworld.gov

Online ordering: http://www.ntis.gov/ordering.htm

Or

Lawrence Livermore National Laboratory

Technical Information Department's Digital Library

http://www.llnl.gov/tid/Library.html 


\title{
Focusing Magnets for HIF based on Racetracks
}

\author{
Nicolai N. Martovetsky and Robert R. Manahan
}

\begin{abstract}
Heavy Ion Fusion (HIF) is considered a promising path to a practical fusion reactor. A driver for a HIF reactor will require a large number of quadrupole arrays to focus heavy ion beams. A conceptual design, and trade off studies of the quadrupole array based on racetracks are presented. A comparison with a conventional shell magnet is given and advantages and disadvantages are discussed. $A$ more detailed design of a single quadrupole for the High Current eXperiment (HCX) is presented and discussed.
\end{abstract}

Index Terms- Quadrupole, racetrack winding, gradient, harmonics

\section{INTRODUCTION}

Tacc N the HIF reactor concept many heavy ion beams are accelerated in a linear accelerator and focused on a target, which ignites and produces fusion energy. A driver for the reactor has to be a reliable and economical device to make the concept a competitive source of energy. The requirements for the focusing power of the driver are very high, which makes the superconducting magnet quadrupoles the most attractive option for focusing the heavy ion beams in the accelerator. The focusing quadrupole magnets have been developed and used successfully in the High Energy Physics (HEP) accelerators at FNAL, DESY, CERN for a long time. However, the new feature in the HIF drivers is that the magnets have to focus many tightly packed beams, which form a regular rectangular array. This main feature and the other features, like less stringent requirements for the field quality, short magnet lengths of the array, and a very strong requirement for a low cost result in a search for the most efficient concept for the focusing arrays.

There are several approaches to the solution of the problem. One approach is to try to utilize the technology developed for HEP for the shell magnets (i.e. when the windings are located on the cylindrical surfaces around the beam tube) and to expand it to the multi-beam arrays. The direct copying of the quadrupoles developed for ring accelerators provides a very good quality field [1]. But there is a concern that this approach may not be a very promising one, mainly from the economic standpoint [2]. An innovative fabrication process for the shell magnets is described in [2] which has a potential to reduce the production cost.

Another approach is to use racetrack windings to create an array, which is quite different from the traditional geometry for the accelerator magnets, but is usual for other applications. The racetracks have a long history of applications in electromotors, generators, wigglers and undulators. In particle accelerators they are used mostly in non-superconducting magnets due to the simplicity of the winding, lower cost and the fact that the contribution to the magnetic field comes mostly from the iron poles, rather from the windings. For the superconducting magnets, where the conductors generate most of the field, the shell type magnets wound on a cylindrical surface were the primary line of development due to a higher efficiency of the superconductor use.

However, lately the racetrack winding has becom an object of growing interest for the HEP colliders after introduction of the dual aperture accelerators [3-4]. The racetrack geometry appears to be very attractive both performance and cost wise, when the magnet flux can be shared between the two apertures. For the HIF focusing array, the flux is shared between many apertures for the beams and the concept of the racetracks seems to be attractive as well.

Another reason for interest towards the racetrack windings is the desire to reduce training and degradation in the superconducting magnets. Training and degradation in the magnets result in inability to reach the designed parameters until after many quenches, and in inability to reach the conductor properties even after many charges. The collared shell magnets have relatively complex structures with very tight tolerances to support the forces and eliminate micro movements of the superconductor, which are responsible for training and degradation. But even the best-developed methods of the winding support do not eliminate training and degradation completely.

There is a hope that due to a simpler magnet structure, the racetrack windings will experience much less degradation and training in comparison with the collared shell magnets.

This paper discusses a concept of the focusing arrays on the basis of the racetracks and discusses advantages and disadvantages of the concept.

\section{HIF FOCUSING ARRAY}

\section{A. Requirements}

Requirements for the HIF driver are still under study and not finalized. Preliminary, the HIF focusing arrays for the induction accelerators should produce a quadrupole field with an integrated error of better than $0.1 \%$ at $80 \%$ of the radius of the beam tube. Also, there should be a very low stray field outside the array, since there are ferromagnetic
Manuscript received September 19, 2000. This Work supported by the US Department of Energy under contract to the Lawrence Livermore National Laboratory (contract No. W-7405-Eng-48.

Both authors are with Lawrence Livermore National Laboratory, Livermore, CA 94550 USA

Author N.M.: telephone: (925) 4224269 , e-mail: martovetsky1@llnl.gov

Author R.M. :telephone: (925) 422 0679, e-mail: manahan1@llnl.gov 


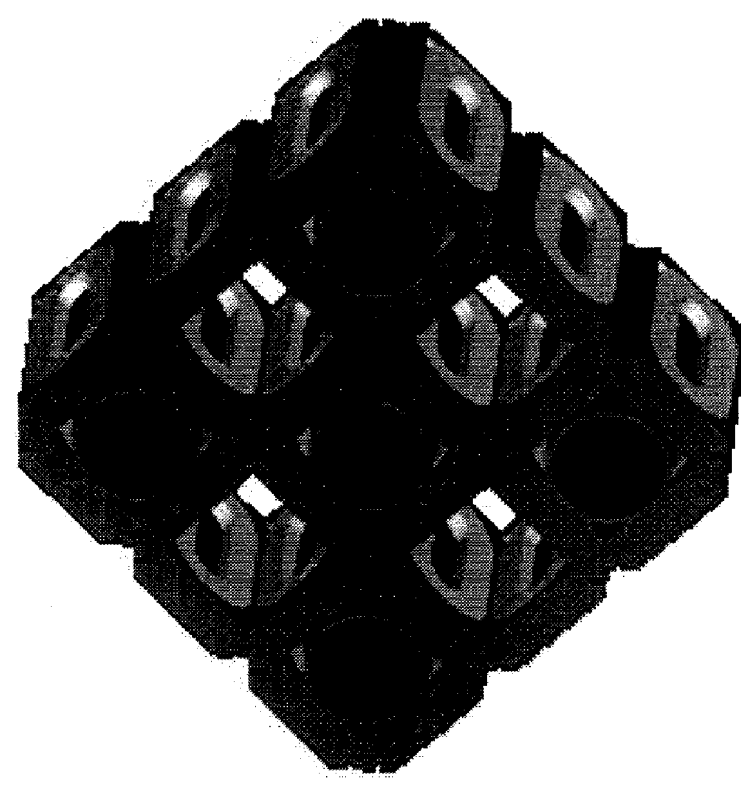

Fig. 1 A $3 \times 3$ fragment of the HIF focusing array

elements in the accelerating sections of the HIF driver. Axially the arrays should have very little wasted space, which requires a shortest possible end part of the racetrack. The maximum field in the winding is foreseen to be in the range of 4-6 $\mathrm{T}$ and the array is required to produce the highest possible magnetic field gradient.

Since several thousands of the arrays will be needed for the HIF driver, one of the most important requirements would be a low production cost of the magnets.

\section{$B$. Conceptual design description}

A view of the $3 \times 3$ fragment of the focusing array with the racetrack windings is shown in Fig.1. Every other beam tube is removed for clarity. The array shown in Fig. 1 is assembled from cells. A cell is assembled from the integrated double-sided racetracks, mounted in the coil forms. The racetracks, which two adjacent cells, are mounted in each coil holder. A single cell of the array is shown in Fig. 2. Such an arrangement makes the interface between neighboring cells much easier than in the shell type arrangement. Because of that simplicity and a very effective use of the structural material to support the electromagnetic forces, the racetrack array can be made very compact.

The array is assembled from identical elements, which makes it very attractive for mass production.

\section{1) Field quality considerations}

To generate a good quality quadrupole field the racetracks are designed to approximate the classical linear current distribution in a square cell. Fig. 3 shows an ideal linear current distribution in a cell of an infinite array which generates a pure quadrupole field and its approximation with the racetrack windings. This deviation from the ideal current distribution creates some error field harmonics, which need to be cancelled out or greatly suppressed. This task is similar to shell magnet design optimization, when appropriate
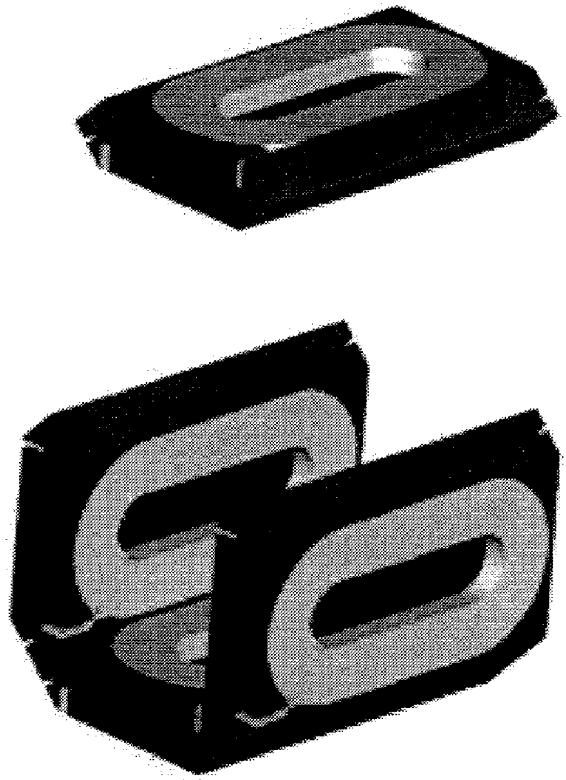

Fig.2. A cell of the quadrupole array and an individual integrated racetrack

spacers allow reducing the error field to an acceptable level. In this respect, racetrack optimization is no more difficult than for shell magnets.

One of the drawbacks of the racetrack current distribution shown in Fig. 3 is that the corners, which ideally should carry the highest current density, are not available for the windings, since the corners are used for the structural interfaces with the neighbors. This problem is not significant for large bore beam tubes $(80-100 \mathrm{~mm}$ diameters), but becomes a factor at smaller apertures.

For small aperture magnets the geometry of the racetrack magnets could be modified to place the conductor in the comers and move the interface between two adjacent racetracks from the comers. That would lead to two basic elements, instead of one, to build a quadrupole cell.

2) Supporting electromagnetic forces

When the array is energized, significant electromagnetic forces are generated, which need to be supported by the structure to prevent possible quenching and change of the geometry, which may distort the field. Fig. 4. Illustrates the forces in the shell type and the racetrack type magnets (one quadrant of the cross section is shown only). The distribution of forces in the magnet in an array is significantly different from that in a stand-alone quadrupole.
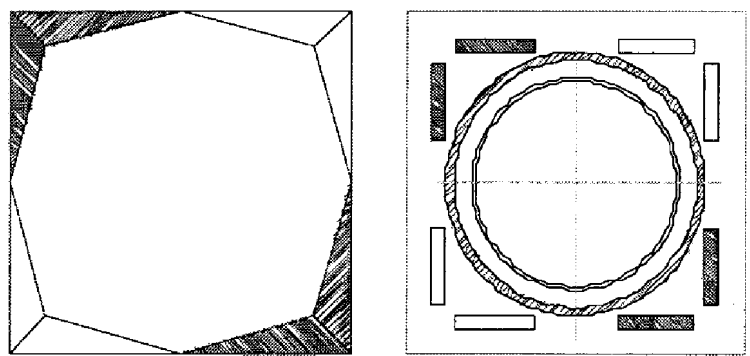

Fig.3. Ideal current distribution in a cell of an infinite array and its approximation with the racetrack windings

In a stand-alone quadrupole, there is a component of the force directed inward (see Fig.4 a and b). A racetrack 
configuration is not very efficient to oppose this component, since the forces tend to separate the winding from the racetrack structure. The shell magnet geometry is very convenient for the force distribution and a hoop structure and the wedges between the winding packs on the collars support these forces very efficiently.

In contrast to the single quadrupole, in the array configuration, the forces generated in the windings are easily supported in the racetrack configuration shown in Fig.2. Due to the symmetry, all out-of-plane forces in the racetrack are cancelled within the double-sided racetrack structure, which makes the coil holder react the in-plane forces only. This is the most efficient use of the structural material.

Also, such a design is convenient for giving a prestress to the winding pack from inside the racetrack, using wedges. Ensuring an adequate prestress is considered one of the most important measures to reduce degradation and training in the coils.

The shell type magnets use collars and/or shells or shrink fit cylinders [2] to contain the electromagnetic forces and to provide a prestress on the winding during assembly. Since utilization of the structural material in the racetrack cell is more efficient, the racetrack configuration can be made more compact.

\section{Comparison between the shell and the racetrack quadrupole arrays}

To compare performance of two different concepts we analyzed three quadrupole cells for infinite HIF array. All cells were assumed to have $120 \mathrm{~mm}$-diameter beam apertures. The shell magnet cell unit occupies $200 \times 200 \mathrm{~mm}$ space. We compare it to two racetrack cells - one with the same size cell of $200 \times 200 \mathrm{~mm}$ and the other with $180 \times 180 \mathrm{~mm}$ size cell. The cross sections of the compared $200 \mathrm{~mm}$ shell and $180 \mathrm{~mm}$ racetrack cells are shown in Fig.5. Table 1 gives

a
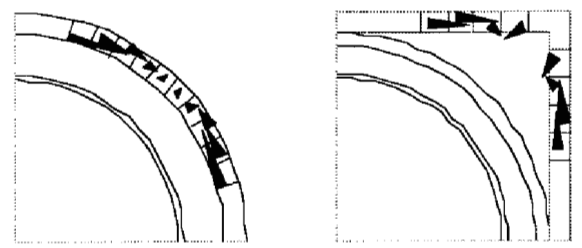

ᄃ

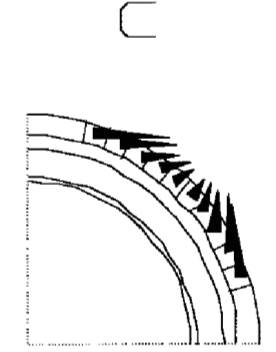

the

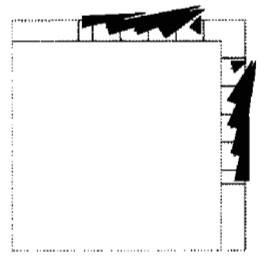

Fig. 4 Forces in the shell magnet cell and a racetrack cell for an infinite array: a) shell magnet single quadrupole, b) racetrack quadrupole, c) shell quad cell in an infinite array, d) racetrack quad cell in an array
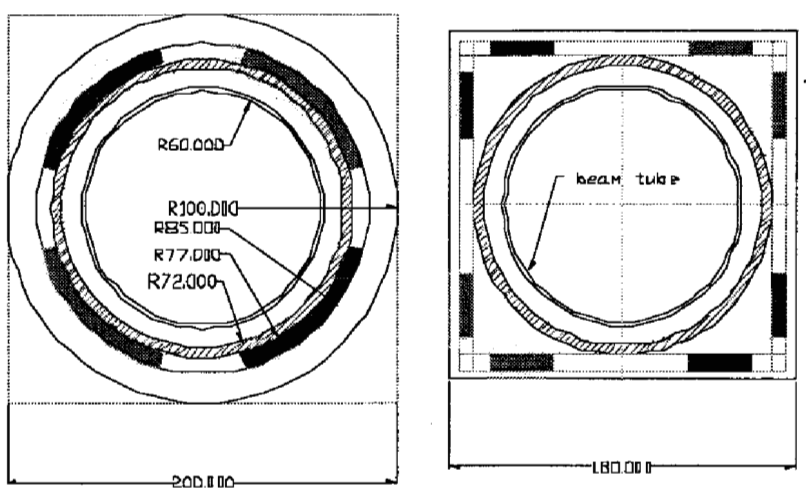

Fig. 5. Comparison between a shell quadrupole and a racetrack quadrupole for infinite array

comparison between the main parameters of the quadrupoles.

For the trade off study we assumed that the thickness of the winding is the same. We designed the windings with no gaps. We selected the peak field in the winding to be around 4.5-5 $\mathrm{T}$. We designed the $2 \mathrm{D}$ geometry for all options in such a way that the main error field component, the dodecapole component a6, would be zero.

TABLE 1. COMPARISON OF THE SHELL AND RACETRACK QUADRUPOLES IN ARRAY

\begin{tabular}{|c|c|c|c|}
\hline $\begin{array}{c}\text { Harmonics } \\
(\mathrm{R}=48 \mathrm{~mm}\end{array}$ & $\begin{array}{c}\text { shell, 200 } \\
\mathrm{mm} \text { cell }\end{array}$ & $\begin{array}{c}\text { Racetrack, } \\
200 \mathrm{~mm} \text { cell }\end{array}$ & $\begin{array}{c}\text { Racetrack, } \\
180 \mathrm{~mm} \text { cell }\end{array}$ \\
\hline $\mathrm{a} 2, \mathrm{~T}$ & 2.20 & 1.73 & 2.01 \\
\hline $\mathrm{a} 10, \mathrm{~T}$ & $-5.75 \mathrm{E}-03$ & $-3.50 \mathrm{E}-03$ & $-4.01 \mathrm{E}-03$ \\
\hline I total, $\mathrm{A}$ & $1.62 \mathrm{E}+05$ & $1.35 \mathrm{E}+05$ & $1.30 \mathrm{E}+05$ \\
\hline Bmax, T & 4.51 & 4.51 & 5.12 \\
\hline $\begin{array}{c}\text { Gradient, } \\
\mathrm{T} / \mathrm{m}\end{array}$ & 45.78 & 36.08 & 41.91 \\
\hline Grad//*1e5 & 28.35 & 26.69 & 32.24 \\
\hline
\end{tabular}

Table 1 shows that in array, the shell magnet with the same cell size is still more efficient than the racetrack cell, since the conductor is closer to the aperture. However, the difference is much smaller than that for a single quadrupole magnet. If a slightly smaller racetrack cell can be built due to a simpler interface and less structure, the performance of the racetrack is very competitive with the shell magnet. The error field can be maintained low and the gradient per unit of superconductor (or current) is even a little higher for the racetrack cell than for the shell cell in our study.

\section{Termination of the infinite array with racetrack windings}

The HIF driver will have 21-100 beams, which translates into $5 \times 5$ to $10 \times 10$ arrays with possible modifications to the corner cells. If nothing is done at the periphery of the array, the outside row of cells will have too poor of a field quality to be used for the beams and this is unacceptably inefficient. To maintain a high quality quadrupole field in all cells of the array, compensation windings have to be used on the periphery of the array. The generic approach to the termination of the finite array to simulate the infinite array is described in [5]. This approach eliminates the stray field outside the magnet and makes the field in all cells purely quadrupole. The implementation of this approach was studied for the shell magnets and it was found that it requires an additional space of half of the cell size around the array and rather complicated geometry windings to simulate an infinite array [5]. Our studies on the example of $3 \times 3$ array 
showed that the racetrack array could be easily terminated with auxiliary racetrack windings to cancel out the stray field and to maintain a high quality field in all the cells of the array. Thus, termination of the quadrupole array is possible without a new type of windings and is easier than termination of the shell magnets array.

\section{E. Conductor selection}

The racetrack quadrupoles have a wide selection of the superconductor. Any conductor used for the shell magnet can be utilized in the racetracks, however from the mechanical standpoint it would be beneficial to use a monolithic conductor with a rectangular cross section. That will allow the creation of a geometrically very well defined winding pack with a high modulus and very low deflections, thus minimizing the probability of a quench. Monolithic conductors are acceptable for HIF application since it is essentially a DC device and AC loss is not a factor. A monolithic conductor can be made from a regular multifilamentary or APC round and twisted conductor by rolling it down to a rectangular cross section. The aspect ratio of the rectangular conductors can be up to $1: 7$ before a noticeable anisotropic effect of $30 \%$ takes place. This anisotropic effect results in the decrease of the critical current density in the conductor when its wider side is oriented perpendicular to the magnetic field [6]. Simultaneously, the critical current increases if the conductor is oriented with its wider side parallel to the magnetic field. In our application this effect of anisotropy is not that essential and may even be beneficial, since the undesirable magnetic field component is present only in the low field areas, while enhancement of the critical current in the parallel high field provides a larger operational margin. Such a conductor is a much more economic option than a traditional Rutherford cable or other cables.

\section{HIGH CURRENT EXPERIMENT (HCX) QUADRUPOLE WITH RACETRACK COILS}

A collaborating team of LBL, PPPL and LLNL is building a High Current Experiment to validate many critical issues for developing a HIF Integrated Research Experiment, which will employ superconducting focusing arrays. The HCX will use a single beam and the Second Phase of the HCX will use the superconducting focusing quadrupole.

To advance the design of the racetrack quadrupole and to study the potentials of the approach we designed a quadrupole for a single beam, and we are in the process of prototype work.

The concept of the racetrack quadrupole for the array is integrated into a single beam quadrupole. Although the theoretical efficiency of the racetracks for a single quadrupole is less than the efficiency of the shell magnets, a high current density in the winding pack and a relative simplicity of the design make the racetrack geometry

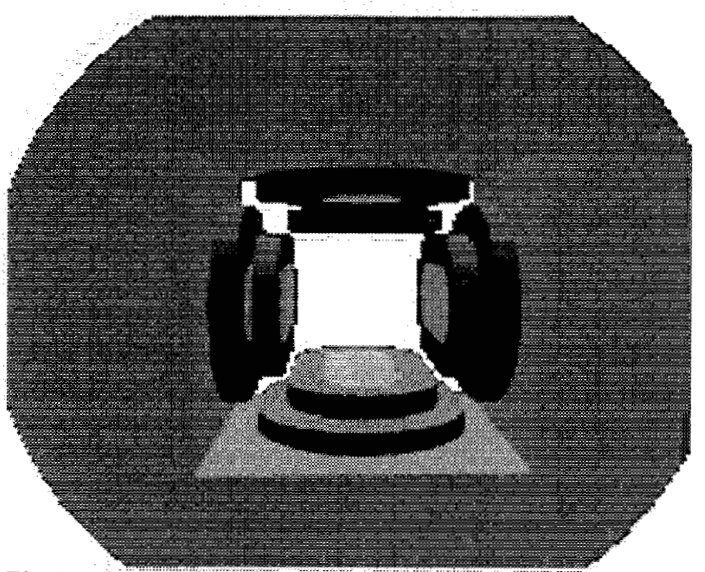

Fig.6. HCX quadrupole with racetrack windings

competitive against the shell type magnets.

The schematic representation of the HCX quadrupole is shown in Fig. 6. In contrast to the array where the ferromagnetic shield does not improve the magnet performance, the iron yoke and the ferromagnetic inserts in the racetrack windings improve the gradient of the quadrupole significantly and bring the efficiency of the racetrack quad closer to the efficiency of the shell magnet [7].

\section{CONCLUSIONS}

The racetrack winding is a promising concept for the HIF focusing arrays due to the following features:

- Efficient support structure and stress management

- Simple interfaces between cells

- Convenient assembly of the array

- Interchangeable modules

- Attractive for mass production ACKNOWLEDGMENT

The authors are grateful to $\mathrm{R}$. Bangerter, S. Lund, P. Seidl, G. Logan and V. Karpenko for useful discussions and support in the development of the concept

\section{REFERENCES}

[1] S. Caspi, R.Bangerter, K.Chow, A.Chow, A.Faltens et al, "Superconducting Quadrupole Magnet Array for a Heavy Ion Fusion Driver," IEEE Trans. Applied Superconductivity, vol. 9, No 2, pp. 463-464, June 1999

[2] R.B. Meinke, A. Faltens, R.O. Bangerter, R.M. Scanlan, P. Seidl"Development of Quadrupole Arrays for Heavy Ion Fusion," IEEE Trans. Applied Superconductivity, vol. 10, No 1, pp. 192-195, March 2000.

[3] S.A.Gourlay, P.Bish, S.Caspi, K.Chow, et al, "Design and Fabrication of a $14 \mathrm{~T}, \mathrm{Nb} 3 \mathrm{Sn}$ Superconducting Racetrack Dipole Magnet", IEEE Trans. Applied Superconductivity, vol. 10, No 1, pp. 294-297, March 2000.

[4] G.Sabbi, G. Ambrosio, N. Andreev, et al, "Conceptual Design of a Common Coil Dipole for VHLC," IEEE Trans. Applied Superconductivity, vol. 10, No 1, pp. 330-333, March 2000.

[5] A.Faltens, D. Shuman, "A Superconducting Quadrupole Array for Transport of Multiple High Current Beams", Proceedings of $18^{\text {th }}$ IEEE SOFE, October 25-29, 1999, Albuquerque, p.352-356.

[6] M.Garber, T. Luhman, W. Sampson, and M. Suenaga, Critical Current Optimisation of Isabelle Braid, Part 1, Anisotropy Effects in Wires and Braids, BNL, Isabelle Project Technical Note No. 315

[7] Richard J. Thome, private communication 